\title{
LIVER FIBROSIS GRADE CLASSIFICATION WITH B-MODE ULTRASOUND
}

\author{
Wen-Chun Yeh, Sheng-Wen Huang and Pai-Chi Li \\ Department of Electrical Engineering, National Taiwan University, Taipei, Taiwan
}

(Received 27 January 2003; revised 15 May 2003; in final form 29 May 2003)

\begin{abstract}
B-mode images of 20 fresh postsurgical human liver samples were obtained to evaluate ultrasound ability in determining the grade of liver fibrosis. Image features derived from gray level concurrence and nonseparable wavelet transform were extracted to classify fibrosis with a classifier known as the support vector machine. Each liver sample subsequently underwent histologic examination and liver fibrosis was graded from 0 to 5 (i.e., six grades total). The six grades were then combined into two, three, four and six classes. Classifications with the extracted image features by the support vector machine were tested and correlated with histology. The results revealed that the best classification accuracy of two, three, four and six classes were $91 \%$, $85 \%, 81 \%$ and $72 \%$, respectively. Thus, liver fibrosis can be noninvasively characterized with B-mode ultrasound, even though the performance declines as the number of classes increases. The elastic constants of 16 samples out of a total of 20 were also correlated with the image features. The Pearson correlation coefficients indicated that the image features are more strongly correlated with the fibrosis grade than with the elastic constant. (E-mail: paichi@ cc.ee.ntu.edu.tw) @ 2003 World Federation for Ultrasound in Medicine \& Biology.
\end{abstract}

Key Words: Tissue characterization, Gray level concurrence, Nonseparable wavelet transform, Support vector machine, Liver pathology, Fibrosis.

\section{INTRODUCTION}

Viral hepatitis, such as hepatitis $\mathrm{B}$ or $\mathrm{C}$, is one of the causes of liver cirrhosis. The virus can induce chronic inflammation of the liver and result in fibrotic changes. The extent of fibrosis in hepatic tissue can range from fibrous expansion in the portal area to cirrhosis (Poynard et al. 1997). The detection of the fibrosis status is particularly important for patients with intermediate fibrosis. With knowledge of the fibrosis status, proper medication can be administered to prevent further inflammation that might lead to cirrhosis and concomitant severe complications. B-mode ultrasonic imaging has been used to investigate liver conditions because a diffuse fibrotic structure might change the characteristics of the ultrasound image (Yang et al. 1988). However, ultrasound's capacity to differentiate among different fibrosis states, including early cirrhosis and intermediate fibrosis of the liver, is quite limited, even when used by an experienced clinician, because the change in the image is frequently very subtle. Thus far, only biopsy has been able accu-

Address correspondence to: Professor Pai-Chi Li, National Taiwan University, Department of Electrical Engineering, No. 1, Sec. 4, Roosevelt Road, Taipei 106, Taiwan. E-mail: paichi@cc.ee.ntu.edu.tw rately to identify the fibrosis state. However, needle biopsy of the liver is invasive and can cause severe complications, though the incidence rate is very low. Therefore, a noninvasive method for detecting the status of the liver is desired.

Several researchers have examined liver tissue classification by using the features of B-mode images. Nicolas at al. (1986) first used B-mode textures to discriminate between the liver and the spleen of a healthy human. Wu et al. (1992) explored the application of multiresolution fractal analysis of hepatoma, cirrhosis and the healthy liver; the accuracy was satisfactory. Kadah et al. (1996) tested various classification algorithms with statistical analysis and neural network methods. The classification technique significantly affected the final diagnosis. Mojsilovic et al. $(1996,1998)$ first presented a $2 \times$ 1-D separable wavelet transform method for detecting early cirrhosis. A nonseparable wavelet decomposition method was then proposed to differentiate among healthy liver, cirrhosis and steatosis. The nonseparable wavelet transform (NSW) method was found to yield an excellent detection rate. Notably, these studies focused only on differentiating a cirrhotic from a healthy liver. The intermediate fibrosis between a cirrhotic and a 
healthy liver has not been detected, though it is associated with chronic active hepatitis. Ogawa et al. (1998) used neural networks to differentiate among chronic active hepatitis, cirrhosis and healthy liver. However, they could not determine the actual fibrosis grade because histologic examination was not performed in all cases.

The main purpose of this study was to investigate the possibility of determining the grade of fibrosis with the use of B-mode image features. Additionally, the support vector machine (SVM), a classification technique proposed by Cortes and Vapnik (1995), was used. The SVM has been applied in other biomedical research areas, such as the detection of delayed gastric emptying from elastogastrograms (Liang et al. 2001) and the diagnosis of glaucoma with standard automated perimetry (Chan et al. 2002), but it has not been applied in medical ultrasonic imaging. In this study, the SVM was employed to classify fibrosis. Performance of the SVM was also compared with that of a more commonly used technique, known as the Bayes classification technique.

This article is organized as follows. The next section described methods of image feature extraction and classification. The image acquisition protocols and the experimental set-up are subsequently described. Classification results are summarized. Finally, the general performance of the SVM and the correlation between the image features and the elastic properties are discussed.

\section{METHODS}

\section{Image feature extraction and classification}

The gray level concurrence (GLC) method and the NSW were used to extract image features. Additionally, the SVM was used as the classifier. In the GLC method, a spatial gray-level dependence matrix (concurrence matrix) is first calculated (Haralick et al. 1973). The matrix, $C o(i, j ; d, \theta)$, represents the probability that the first pixel has a gray-level of $i$ and the second pixel has a gray-level of $j$ under the condition that the distance between two pixels is $d$ in the direction of $\theta$. For example, the following equation indicates the concurrence matrix at $\theta=$ $0^{\circ}$. The concurrence matrix at other angles can be similarly defined (Wu et al. 1992).

$$
\begin{aligned}
& \operatorname{Co}\left(i, j ; d, 0^{\circ}\right) \\
& \quad=\#\left\{\begin{array}{l}
((k, l),(m, n)) \in\left(L_{y} \times L_{x}\right) \\
k=m,|l-n|=d \\
I(k, l)=i, I(m, n)=j
\end{array}\right\} / T\left(d, 0^{\circ}\right)
\end{aligned}
$$

where $(k, l)$ and $(m, n)$ are coordinates of two pixels in an image; the image has a size of $L_{y} \times L_{x}$ and $I$ represents the intensity of a particular pixel. The symbol $\#\{\cdot\}$ denotes the total number of occurrences and $T$ is the total number of pixel pairs with the prespecified $d$ and $\theta$.

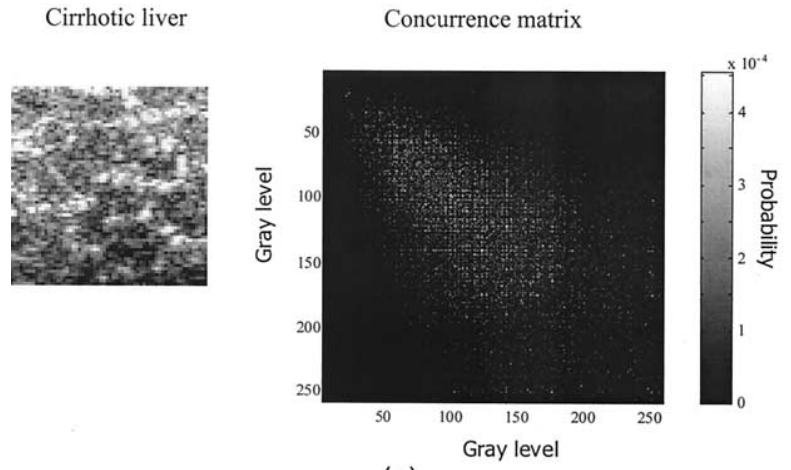

(a)

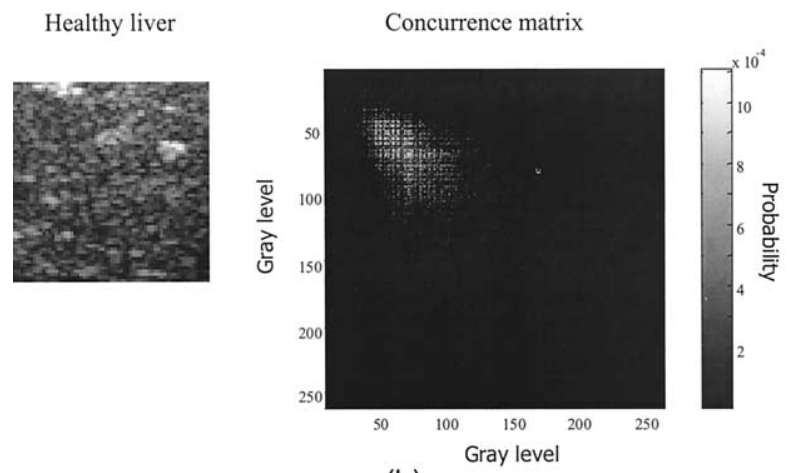

(b)

Fig. 1. B-mode image and its corresponding concurrence matrix of (a) A cirrhotic liver and (b) A healthy liver.

Figures $1 \mathrm{a}$ and $1 \mathrm{~b}$ show the concurrence matrices $(d=6$, $\theta=0^{\circ}$ ) for images of a cirrhotic liver and a healthy liver, respectively. Generally, the energy of the concurrence matrix of a healthy liver is more concentrated than that of a cirrhotic liver.

Image features such as the angular second moment, contrast, correlation, entropy and sum entropy can be derived from the concurrence matrix. They are defined as follows.

a) Angular second moment: $\sum_{i, j \in G}[C o(i, j ; d, \theta)]^{2}$.

b) Contrast: $\sum_{i, j \in G}(i-j)^{2} \cdot C o(i, j ; d, \theta)$.

c) Correlation: $\frac{\sum_{i, j \in G} i j \operatorname{Co}(i, j ; d, \theta)-m_{x} \cdot m_{y}}{S_{x} \cdot S_{y}}$, where $m_{x}=\sum_{i} i \sum_{j} C o(i, j ; d, \theta)$, $m_{y}=\sum_{j} j \sum_{i} \operatorname{Co}(i, j ; d, \theta)$ 
Table 1. Filter coefficients for the three possible pairs of the filters

\begin{tabular}{|c|c|c|c|c|c|c|c|}
\hline \multirow[b]{2}{*}{ 2-D diamond-shaped filter } & \multirow[b]{2}{*}{ Pair } & \multicolumn{2}{|c|}{ A } & \multicolumn{2}{|c|}{ B } & \multicolumn{2}{|c|}{$\mathrm{C}$} \\
\hline & & LP & $\mathrm{HP}$ & LP & HP & LP & HP \\
\hline h4 & ho & 0.875 & 0.5 & 0.6879 & 0.5863 & 0.65 & 0.6607 \\
\hline$h 7 h 3 h 7$ & hl & 0.125 & -0.125 & 0.1398 & -0.1649 & 0.125 & -0.1344 \\
\hline$h 8 h 6 h 2 h 6 h 8$ & $h 2$ & -0.0313 & 0 & -0.0196 & -0.0072 & -0.0125 & -0.0134 \\
\hline$h 7$ h6 h5 h1 h5 h6 h7 & $h 3$ & 0 & 0 & -0.0021 & 0.0057 & 0 & 0.0013 \\
\hline h4 h3 h2 h1 h0 h1 h2 h3 h4 & h4 & 0 & 0 & 0.0017 & 0 & 0 & 0 \\
\hline$h 7$ h6 h5 h1 h5 h6 h7 & h5 & -0.0625 & 0 & -0.0525 & -0.0144 & -0.025 & -0.0268 \\
\hline$h 8 h 6 h 2 h 6 h 8$ & h6 & 0 & 0 & -0.0063 & 0.0171 & 0 & 0.004 \\
\hline$h 7 h 3 h 7$ & $h 7$ & 0 & 0 & 0.0067 & 0 & 0 & 0 \\
\hline h4 & $h 8$ & 0 & 0 & 0.01 & 0 & 0 & 0 \\
\hline
\end{tabular}

$h 0$ to $h 8=$ filter coefficients; $\mathrm{LP}=$ low-pass filter; HP $=$ high-pass filter.

$$
\begin{aligned}
& S_{x}{ }^{2}=\sum_{i} i^{2} \sum_{j} C o(i, j ; d, \theta)-m_{x}{ }^{2}, \\
& S_{y}{ }^{2}=\sum_{j} j^{2} \sum_{i} C o(i, j ; d, \theta)-m_{y}{ }^{2} .
\end{aligned}
$$

d) Entropy: $-\sum_{i, j \in G} \operatorname{Co}(i, j) \cdot \log [C o(i, j ; d, \theta)]$.

e) Sum entropy: $-\sum_{k} M_{x+y}(k) \cdot \log \left[M_{x+y}(k)\right]$,

$$
\text { where } M_{x+y}(k)=-\sum_{\substack{i \\ i+j=k}} \sum_{j} C o(i, j ; d, \theta) .
$$

Note that $G$ in these equations represents the set of available gray-level values in the digitized image. In this work, the images were digitized with 8-bits, so the possible gray levels ranged from 0 to 255 . Generally, the angular second moment is the second moment of the concurrent matrix. Contrast weights the concurrence matrix with the square of the difference between two gray levels. Entropy yields the energy distribution of the concurrence matrix. Sum entropy first produces an array of sums of $C o(i, j ; d, \theta)$, where the sum of two pixel gray levels are at a prespecified value $k$, and then calculates the entropy of the array. The liver images can be characterized with these features derived from GLC.

In addition, NSW was also applied to extract the features of images. The quincunx wavelet transform was applied (Mojsilovic et al. 1998). In this case, the original image was filtered by a high-pass filter and a low-pass filter before it was down-sampled to a new image with half of the original area. Let $(i, j)$ be the pixel index of an image with a size of $L_{x} \times L_{y} ; 0 \leq i \leq x$ and $0 \leq j \leq y$. The original image is down-sampled such that only the pixels for which $(i+j)$ is even remain. Table 1 presents the filter coefficients of three possible 2-D diamondshaped high-pass/low-pass filter pairs. Filtering and down-sampling were performed four times. Figure 2 depicts such a process for a typical cirrhotic liver image.
Note that the down-sampled image is rotated counterclockwise such that a square becomes a diamond, and vice versa. The variances of images $\mathrm{H} 1$ to $\mathrm{H} 4$ and $\mathrm{L} 4$ were taken as the image features (Mojsilovic et al. 1998). Figure 3 compares the NSW output images of a cirrhotic liver (a) to those of a healthy liver (b). In this particular case, the variances of the output images ( $\mathrm{H} 1$ to $\mathrm{H} 4$ and L4) of a cirrhotic liver (198.2, 479.8, 426.2, 810.8 and 2916.2, respectively) exceed those of a healthy liver (43.6, 126.2, 148.8, 292.7 and 995.3, respectively).

The features extracted by the preceding two methods are used for classification. Implementation of the classifier involves a training process that uses some of the data and a testing process that uses the rest. Commonly used classification methods include the Bayes minimum-error classifier (Mojsilovic et al. 1998), the neural network classifier (Ogawa et al. 1998) and the SVM classifier (Chan et al. 2002). This study used the SVM. The aim of the SVM is to find a decision plane that has a maximum distance (margin) from the nearest train-

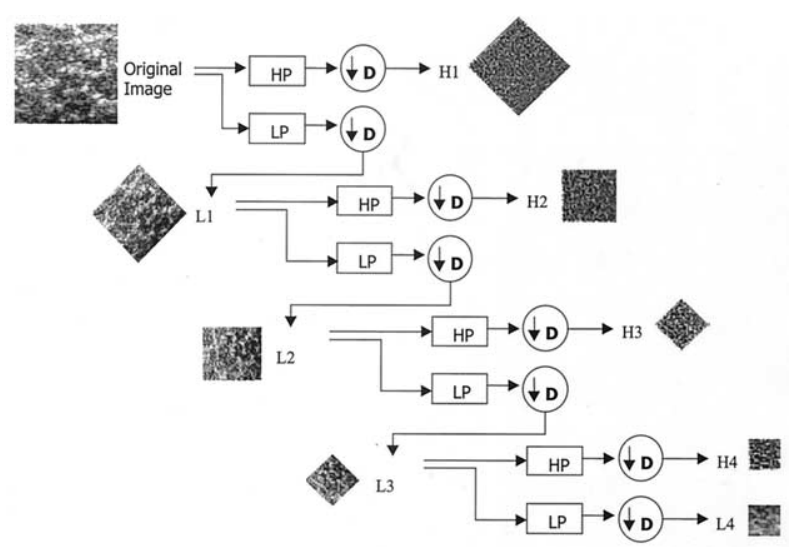

Fig. 2. Nonseparable wavelet transform. HP = high-pass filter; $\mathrm{LP}=$ low-pass filter; $\mathrm{D}=$ down-sampling; $\mathrm{H} 1$ to $\mathrm{H} 4=$ images after high-pass filtering and down-sampling; L1 to L4 = images after low-pass filtering and down-sampling. 
(a)

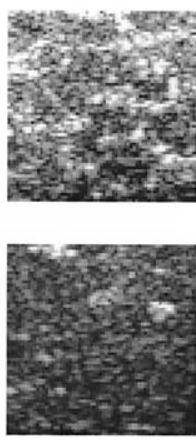

Original image
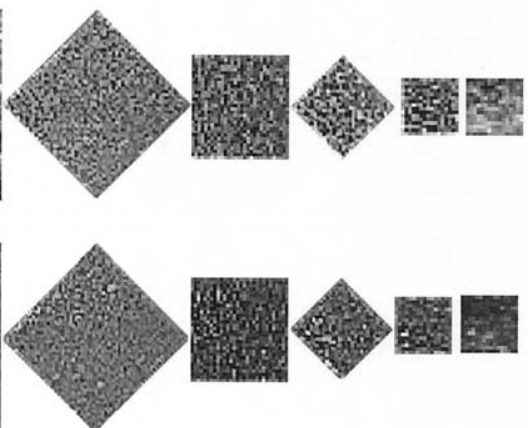

$\mathrm{H} 3 \quad \mathrm{H} 4 \quad \mathrm{~L} 4$
Fig. 3. Nonseparable wavelet transform of (a) A cirrhotic liver (fibrosis grade 4) and (b) A healthy liver (fibrosis grade 0). H1 to $\mathrm{H} 4$ = images after high-pass filtering and down-sampling; L4 = image after final low-pass filtering and down-sampling.

ing pattern. Given the training data $\left\{\left(x_{i}, y_{i}\right) \mid y_{i}=1\right.$ or $-1, i=1, \ldots, N\}$ for a two-class classification (where $x_{i}$ is the input feature; $y_{i}$ is the class label and $N$ is the number of training samples), the SVM maps the features to a higher-dimensional space with a function $\phi$, such that $z=\phi(x)$. The SVM then finds a hyperplane to separate the two classes with the decision boundary set by the support vectors (Burges 1998). The general form of the decision function $\mu(x)$ for the SVM is

$$
\mu(x)=\sum_{i=1}^{N} \alpha_{i} y_{i} z_{i}^{T} z+b,
$$

where the $\alpha_{i} \mathrm{~s}$ and $b$ are selected during the training process, constrained by $\Sigma \alpha_{i} y_{i}=0$ and $0 \leq \alpha_{i} \leq A$, where $A$ is a user-defined penalty term (cost of constraint violation), regulating the general performance of the SVM. Under certain conditions, the computationally intensive mapping process can be reduced with an appropriate kernel function $K$ such that the decision function $\mu(x)$ becomes

$$
\mu(x)=\sum_{i=1}^{N} \alpha_{i} y_{i} K\left(x_{i}, x\right)+b .
$$

Upon training, only a fraction of the $\alpha_{i}$ terms are nonzero. For those $\alpha_{i}$ s that are nonzero, the corresponding training examples must lie nearest to the margins of the decision boundary and these examples are called support vectors (Burges 1998, Chan et al. 2002). The three-class SVM classifier can be generated from a two-class classifier by further dividing one of the original classes into two classes. Higher classes of classifier can be produced similarly.

A multiclass SVM classifier, developed by Junshui Ma and Yi Zhao of Ohio State University, was used

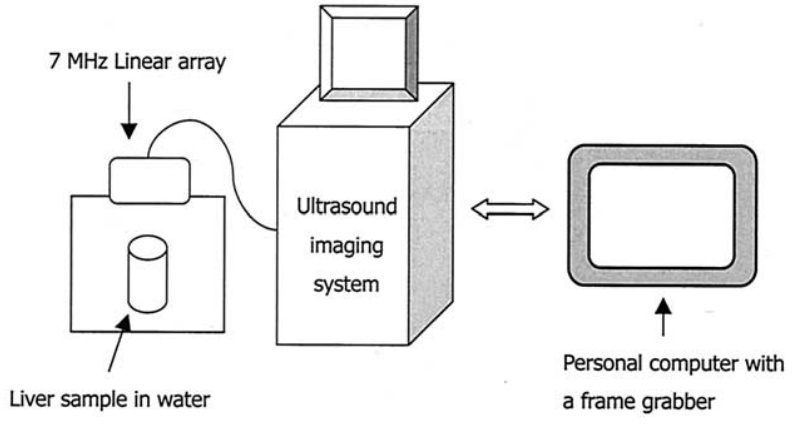

Fig. 4. Set-up for image acquisition.

(Chang and Lin 2001). The two kernel functions used are listed below:

$$
\text { Polynomial: } K\left(x_{i}, x\right)=\left(x_{i}^{T} x+1\right)^{P} \text {, }
$$

Gaussian radial-basis function: $K\left(x_{i}, x\right)=e^{-\gamma\left|x_{i}-x\right|^{2}}$,

where $P$ is the degree of the polynomial kernel and $\gamma$ is the coefficient of the Gaussian radial basis function kernel. The input feature parameters were normalized to between zero and one before training.

\section{Image acquisition and processing}

Twenty fresh human liver samples obtained from surgical specimens were studied. These specimens were 3 to $5 \mathrm{~cm}$ wide and generally had a cubic shape. For some specimens, however, the nontumor part was near the edge of the liver and, thus, one of the cut surfaces of the liver specimen became triangular and the thickness might also have been reduced. The scanning positions were changed such that different B-mode images (10 to 15 images for each sample) with low correlation among each other could be acquired. Thus, the shape of the specimen in the raw image might or might not be rectangular. Nonetheless, only one subimage (with a square shape) was selected from each raw image for signal processing. The images were acquired with a frame grabber (UPG401B, UPMOST Corp., Taipei, Taiwan) with 256 gray levels and $640 \times 480$ pixels. Figure 4 shows the setup for image acquisition.

The original images acquired by the frame grabber (i.e., $640 \times 480$ pixels) corresponded to a size of $15 \mathrm{~cm}$ $\times 11 \mathrm{~cm}$. Note that the original image contains both the liver and the surrounding water. The size of the liver specimen was generally around 3 to $4 \mathrm{~cm} \times 3$ to $4 \mathrm{~cm}$. Thus, in each image, only one subimage of $64 \times 64$ pixels (approximately $1.5 \mathrm{~cm} \times 1.5 \mathrm{~cm}$ ) was selected to ensure that the subimage only contained information from the specimen. Areas of water, blood vessels and 
Table 2. The number of images in each fibrosis grade

\begin{tabular}{lccccccc}
\hline & \multicolumn{6}{c}{ Fibrosis grade } & \\
\cline { 2 - 7 } & 0 & 1 & 2 & 3 & 4 & 5 & Total \\
\hline $\begin{array}{c}\text { Number of } \\
\text { images }\end{array}$ & 10 & 5 & 15 & 20 & 35 & 15 & 100 \\
\hline
\end{tabular}

image artifacts, including shadowing, were avoided. For each specimen, on the other hand, 10 to 15 images from different scanning positions were acquired and only five subimages were selected. These five subimages had low correlation coefficients (less than 0.5) among each other, effectively to increase the sample size and reduce the estimation bias. Gray-scale mapping was adjusted individually, such that the entire display dynamic range was fully used.

The methods of feature extraction, described in the preceding section, were applied to these subimages. After the B-mode images were obtained, the specimens were fixed in formalin and embedded in paraffin. Sections with a thickness of $5 \mu \mathrm{m}$ were cut and stained with hematoxylin and eosin for histologic examination. Fibrosis scores were assigned according to the criteria of the METAVIR Cooperative Study Group (Poynard et al. 1997). Liver fibrosis was originally scored from 0 to 4 as follows: 0: no fibrosis; 1: portal fibrosis without septa; 2 : septa fibrosis; 3: numerous septa without cirrhosis; and 4: cirrhosis. In cirrhotic livers, the widths of fibrotic bands varied and the score was further divided, such that a score of 4 indicated thin fibrotic bands and a score of 5 indicated thick fibrotic bands (Yeh et al. 2002). Table 2 lists the number of images in each fibrosis grade.

The images were divided into two, three, four or six classes according to the following criteria: two classes, fibrosis 4 to 5 and fibrosis 0 to 3 , representing cirrhosis and noncirrhosis; three classes, fibrosis 4 to 5 , fibrosis 1 to 3 and fibrosis 0 , representing cirrhosis, noncirrhotic fibrosis and healthy liver; four classes (fibrosis 5, fibrosis 4 , fibrosis 1 to 3 and fibrosis 0 ) and six classes (all fibrosis grades). The classification accuracy was tested with the SVM by the leave-one-out method (Weiss 1991, Ney et al. 1995). The leave-one-out method is a particular cross-validation method and can be applied when the sample size is small. It requires that one image is selected for testing while the other images are used for training. After testing, the classification result (correct or not) is recorded. The process is repeated until all images have been tested. The accuracy is then determined. The accuracy is defined as

$$
\text { Accuracy }=\frac{C I}{N}
$$

Table 3. The classification accuracy

\begin{tabular}{|c|c|c|c|c|c|c|c|}
\hline \multirow[b]{3}{*}{ Group } & \multicolumn{7}{|c|}{ Accuracy $(\%)$} \\
\hline & \multicolumn{4}{|c|}{$\operatorname{GLC}(d=6)$} & \multicolumn{3}{|c|}{ NSW } \\
\hline & 1 & 2 & 3 & 4 & A & B & $\mathrm{C}$ \\
\hline 2 & 90 & 81 & 81 & 84 & 77 & 87 & 88 \\
\hline 3 & 82 & 74 & 76 & 79 & 71 & 78 & 76 \\
\hline 4 & 76 & 69 & 72 & 71 & 71 & 75 & 72 \\
\hline 6 & 67 & 53 & 58 & 58 & 56 & 57 & 56 \\
\hline
\end{tabular}

GLC = gray level concurrence; NSW $=$ nonseparable wavelet transform; $\mathrm{d}=$ distance of pixels. $1: \theta=0^{\circ}, 2: \theta=45^{\circ}, 3: \theta=90^{\circ}$ and 4: $\theta=135^{\circ}$ for the GLC; A, B, C: different filter pairs for the NSW, as defined in Table 1.

where $C I$ is the number of correct incidences in a particular class and $N$ is the total number of samples in that class. The penalty term $(A)$ and the kernel function parameters $(P$ and $\gamma)$ were optimized to maximize the accuracy. Additionally, four angles $\left(0^{\circ}, 45^{\circ}, 90^{\circ}\right.$ and $135^{\circ}$ ) and six distances ( $\mathrm{d}=1$ to 6$)$ for GLC and the three filter pairs for NSW (pairs A, B and C in Table 1) were all tested. Various combinations of the parameters were considered to find the best performance achievable.

\section{RESULTS}

Table 3 presents the classification accuracy obtained with the GLC features and the NSW features. Notably, all the classification results herein were estimated with a Gaussian radial-basis function kernel, because this outperformed the polynomial kernel. Table 3 shows that the accuracy of the GLC is similar to that of the NSW. Combining features further improves the performance. The combination of all features of the GLC $(\mathrm{d}=6, \theta=$ $0^{\circ}$ in eqns (2) to (6)) and one feature of the NSW (the H2 feature of the filter pair B) yielded the best results of all the different combinations. The overall accuracy of the classifications with two, three, four and six classes are $91 \%, 85 \%, 81 \%$ and $72 \%$, respectively, as shown in Table 4. Such results are better than those with only the GLC or the NSW features. Figure 5 presents relevant comparisons.

Table 4. The classification accuracy with combined features

\begin{tabular}{cccccccc}
\hline & \multicolumn{6}{c}{ Accuracy in different fibrosis grade (\%) } \\
\cline { 2 - 8 } $\begin{array}{c}\text { Number of } \\
\text { classes }\end{array}$ & 5 & 4 & 3 & 2 & 1 & 0 & Overall \\
\hline 2 & & 90 & & & 92 & & 91 \\
3 & & 90 & & 85 & & 60 & 85 \\
4 & 80 & 82.9 & & 85 & & 60 & 81 \\
6 & 80 & 88.6 & 60 & 53.3 & 100 & 40 & 72 \\
\hline
\end{tabular}




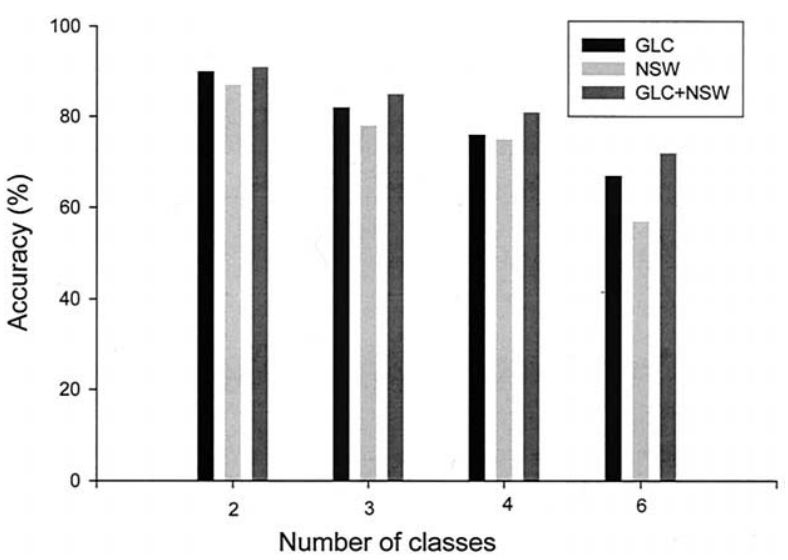

Fig. 5. Comparison of the classification results among the gray level concurrence (GLC) $(d=6, \theta=0)$, the nonseparable wavelet transform (NSW) (filter pair B) and the combination of these two.

\section{DISCUSSION AND CONCLUSIONS}

This work shows that the SVM classifier can detect the different liver fibrosis grades. Combinations of the GLC features and the NSW features yielded the best classification performance. However, the overall accuracy declined as the number of classes increased. As shown in Table 4, the overall accuracy falls to $72 \%$ in six-class classification. One of the reasons for these results is that the number of samples in some cases was small when the number of classes was large. For the class of fibrosis score 0, the accuracy is $60 \%$ when the number of classes was 3 or 4 and the accuracy decreased to $40 \%$ when the number of classes increased to 6 . The classes for fibrosis scores 2 and 3 in the six-class classification also have lower accuracy due to the decrease in the sample size in each class, as shown in Table 4. In other words, the estimation errors increase when the training samples are divided into more classes. Certainly, more samples are desired to increase the accuracy of the classification results.

Previous studies have used transabdominal wall scanning and correlated the images with the pathologic findings obtained with random needle biopsy (Kadah et al. 1996, Mojsilovic et al. 1998). Such a process does not guarantee that the sample obtained in the biopsy is near the image region. Thus, this study has two advantages over previous studies. First, the liver tissues were immersed in water and a 7.0-MHz linear array transducer was used for scanning. Without interference from the abdominal wall, these Bmode images were generally of a higher quality than those obtained in routine clinical practice. The primary difference between our in vitro study and the in vivo studies done by others is the distortion caused by the abdominal wall. Tissue inhomogeneities, particularly from the fat layer, might degrade image quality and affect the classification results. In

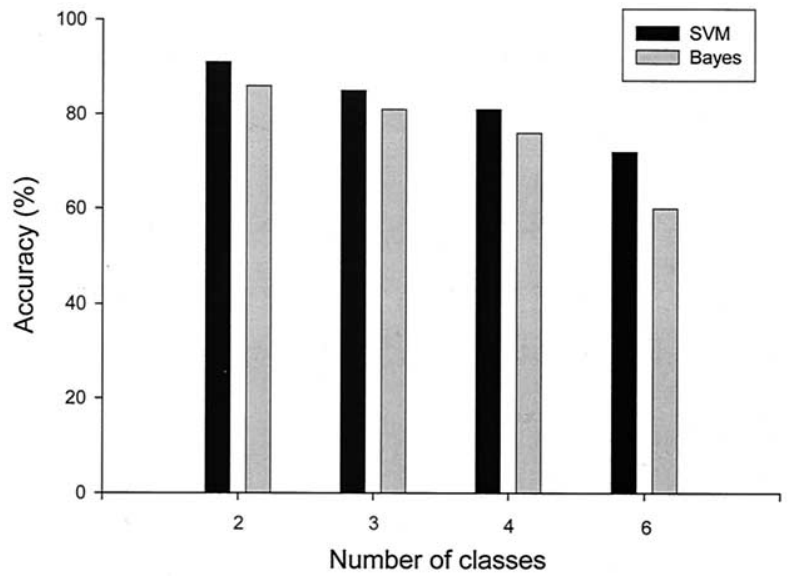

Fig. 6. Comparison between the support vector machine (SVM) classifier and the Bayes classifier.

addition, the images in this study were acquired at $7 \mathrm{MHz}$, which is higher than the typical imaging frequency in clinical situations. The higher frequency might also result in more image details and consequently affect the classification results. Nonetheless, such differences can be reduced with the use of advanced imaging techniques that can reduce the image distortion (e.g., tissue harmonic imaging) and increase the imaging frequency (e.g., coded excitation). Second, the central part of each sample was histologically examined to determine the fibrosis grade. The selected image region was very close to the histologically examined region. Accordingly, the results of the pathologic examination were more closely related to the underlying condition of the liver than in other studies.

Kadah et al. (1996) revealed that the classification technique significantly affected the final diagnosis. The same data set was also tested with a different type of classifier, the Bayes minimum-error classifier, defined as follows (Mojsilovic et al. 1998).

$$
d_{b}(x, i)=\left(x-\mu_{i}\right)^{T} C_{i}^{-1}\left(x-\mu_{i}\right)+\log \left[\operatorname{det}\left(C_{i}\right)\right],
$$

where: $d_{b}$ represents the Bayes distance; $x$ is the input feature vector, $i$ is the class index; $\mu_{i}$ is the mean of the feature vector $i$ and $C_{i}$ is the covariance matrix of the feature vector $i$. The classification is performed by finding the index $i$ that minimizes the Bayes distances. Restated,

$$
d_{b}(x, i)=\min _{i=1, \ldots, N}\left\{d_{b}(x, i)\right\},
$$

where $N$ is the number of classes. The SVM classifier outperformed the Bayes classifier. Figure 6 presents a comparison. 


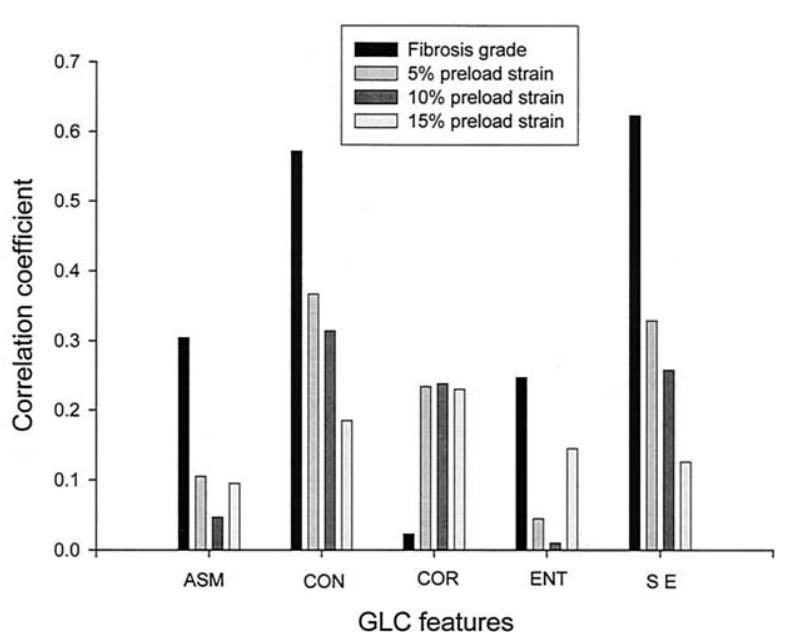

(a)

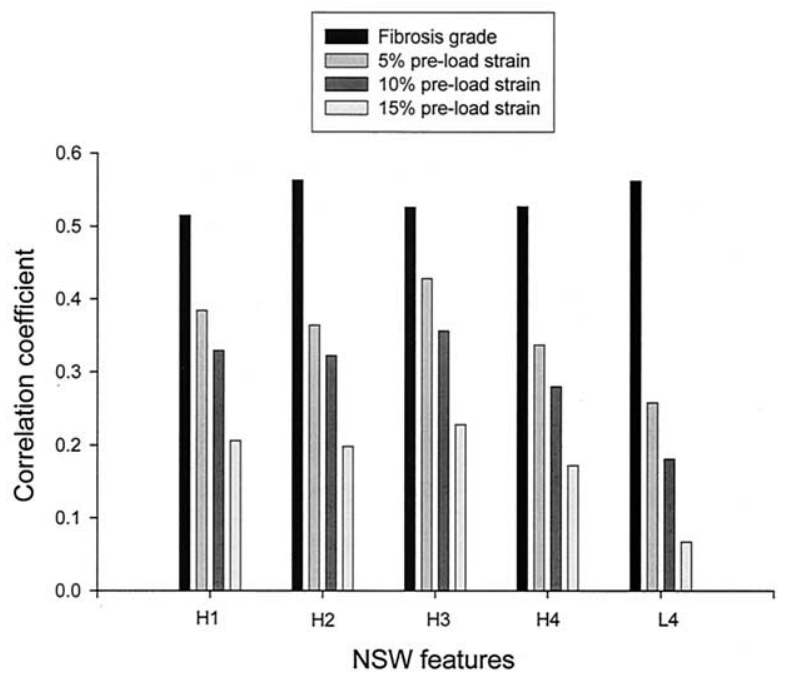

(b)

Fig. 7. (a) Comparison of correlation coefficients between the gray level concurrence (GLC) features and the fibrosis grade, with those between the GLC features and the elastic modulus under different preload strains. ASM $=$ angular second moment; $\mathrm{CON}=$ contrast COR = correlation; ENT = entropy; $\mathrm{SE}=$ sum entropy. (b) Comparison of correlation coefficients between the NSW features and the fibrosis grade, with those between the NSW features and the elastic modulus under different preload strains. $\mathrm{H} 1$ to $\mathrm{H} 4=$ images after high-pass filtering and down-sampling; L4 = image after final low-pass filtering and down-sampling.

The elastic properties of 16 of the 20 liver samples used in this study were also measured in an earlier study (Yeh et al. 2002). The correlation coefficients between the image features and fibrosis grade were compared with those between the image features and the elastic modulus, under $5 \%, 10 \%$ and $15 \%$ preload strains. The correlation coefficients were calculated with the Pearson correlation method (Norusis 1993). As depicted in Fig. 7, the image features are more strongly correlated with the fibrosis grade than with the elastic modulus. Additionally, the correlation coefficient declined as the preload strain increased in most cases. One reason might be that the correlation between the elastic modulus and the fibrosis grade decreases as the strain increases, as indicated by Yeh et al. (2002). As well as examining the B-mode image features and elastic properties, the longterm goal of this study is to investigate further the potential of using various ultrasonic techniques for diagnosing liver fibrosis status.

Acknowledgments-The authors thank the reviewer for the comments. This research was financially supported by the National Science Council under grant no. NSC 92-2213-E-002-019.

\section{REFERENCES}

Burges CJC. A tutorial on support vector machines for pattern recognition. Data Mining Knowledge Discovery 1998;2:955-974.

Chan K, Lee TW, Sample PA, et al. Comparison of machine learning and traditional classifiers in glaucoma diagnosis. IEEE Trans Biomed Eng 2002;49:963-974.

Chang CC, Lin CJ. Training $\nu$-support vector classifiers: Theory and algorithms. Neural Comput 2001;13:2119-2147.

Cortes C, Vapnik V. Support vector networks. Machine Learning 1995;20:273-297.

Haralick RM, Shanmugan K, Dinstein I. Texture features for image classification. IEEE Trans Syst Man Cybernetics 1973;SMC-3: 610-621.

Kadah MY, Farag AA, Zurada MJ, et al. Classification algorithms for quantitative tissue characterization of diffuse liver disease from ultrasound images. IEEE Trans Med Imaging 1996;15:466-478.

Liang H, Lin Z. Detection of delayed gastric emptying from electrogastrograms with support vector machine. IEEE Trans Biomed Eng 2001;48:601-604.

Mojsilovic A, Popovic M, Markovic S, Krstic M. Characterization of visually diffuse diseases from B-scan liver images using non-separable wavelet transform. IEEE Trans Med Imaging 1998;17:541-549.

Mojsilovic A, Popovic M, Sevic D. Classification of the ultrasound liver images with the $2 \times 1$-D wavelet transform. IEEE Int Conf Image Processing 1996;1:367-370.

Ney H, Essen U, Kneser R. On the estimation of 'small' probability by leave-one-out method. IEEE Trans Pattern Anal Machine Intell 1995;17:1202-1212.

Nicolas D, Nassiri DK, Gaarbutt P, Hill CR. Tissue characterization from ultrasound B-scan data. Ultrasound Med Biol 1986;12:135143.

Norusis MJ. SPSS for Windows: Base system user's guide, release 6.0 Chicago: SPSS, 1993.

Ogawa K, Fukushima M, Kubota T, Hisa N. Computer-aided diagnostic system for diffuse liver disease with ultrasonography by neural network. IEEE Trans Nucl Sci 1998;45:3069-3074.

Poynard T, Bedossa P, Opolon P. Natural history of liver fibrosis progression in patients with chronic hepatitis C. The OBSVIRC, METAVIR, CLINIVIR, and DOSVIRC groups. Lancet 1997;349: 825-832.

Weiss SM. Small sample error rate estimation for $k$-NN classification. IEEE Trans Pattern Anal Machine Intell 1991;13:285-289.

Wu C, Chen Y, Hsieh K. Texture features for classification of ultrasonic liver images. IEEE Trans Med Imaging 1992;11:141-152.

Yang PM, Huang GT, Lin JT, et al. Ultrasonography in the diagnosis of benign diffuse parenchymal liver disease: A prospective study. J Formosan Med Assoc 1988;187:966-977.

Yeh WC, Li PC, Jeng YM, et al. Elastic modulus measurements of human liver and correlation with pathology. Ultrasound Med Biol 2002;28:467-474 